\title{
Identifying and Analyzing the Correlations and Inter-Causalities of the Root Causes of Construction Defects
}

\author{
H. Aljassmi, J. Perera, and S. Han
}

\begin{abstract}
Understanding defect causalities is indispensable to its prevention. This paper aims to identify the correlations and inter-causalities amongst the root causes of construction defects, so as to obtain insights about the complex mechanics of defect causation and assist in developing effective defect prevention strategies. Data was collected through a questionnaire survey of 106 professionals in the construction industry. Correlations and inter-causalities analysis showed that time pressure, financial constraints and organizational culture were the most influential root causes.
\end{abstract}

Index Terms-Defect, error, failure, root cause.

\section{INTRODUCTION}

A construction defect is "failing or shortcoming in the function, performance, statutory or user requirements of a building" [1]. Defect costs ranged from $2 \%$ to $6 \%$ of construction costs [2]. Thus, if projects are to succeed, it is imperative to prevent defects. Defect prevention necessitates two major stages: a qualitative and a quantitative stage. The qualitative stage involves systemically identifying and classifying the various causes of defects [3], [4]. On the other hand, the quantitative stage involves observing the most important causes so as to improve aspects of the system that are most capable of restraining defects' recurrence [5]. This paper aims to extract the major causes resulting in defects from extensive literature review; and subsequently conduct a survey with industry practitioners to confirm and revise the causes and then analyze their correlations and inter-causalities.

\section{The Mechanics of Defects}

To prevent construction defects, one must first identify and recognize where these originates. A root cause is the most basic reason for an undesirable condition or problem [6]. If the root cause of the problem is not identified, then one is merely addressing the symptoms and the problem will continue to exist. For this reason, identifying and eliminating root causes of problems is of utmost importance [7], [8]. In order to identify the root causes of construction defect, the Swiss Cheese Model [9] was utilized. Based on the Swiss

Manuscript received February 9, 2013; revised May 9, 2013.

H. Aljassmi and J. Perera are with the School of Civil and Environmental Engineering, University of New South Wales, Sydney, Australia (e-mail: jassmi@me.com; Jivanka@yahoo.com).

$\mathrm{S}$. Han is with the Department of Architectural Engineering, University of Seoul, Korea (e-mail: swhan@uos.ac.kr).
Cheese model, defect causes can be traced back to any of the four descending layers of a system (Fig. 1). The first three layers (Organizational Influences, Defective Supervision, and Preconditions for Defective Acts) represent the root causes and the fourth layer (Defective acts) represents the direct causes. The root causes are also called latent conditions created by higher echelons of the organization owing the emplacement of risky decisions, practices and circumstances. Since the Defective Acts have already being identified in the authors' recent study [4], this study will complementarily focus on identifying the latent conditions.

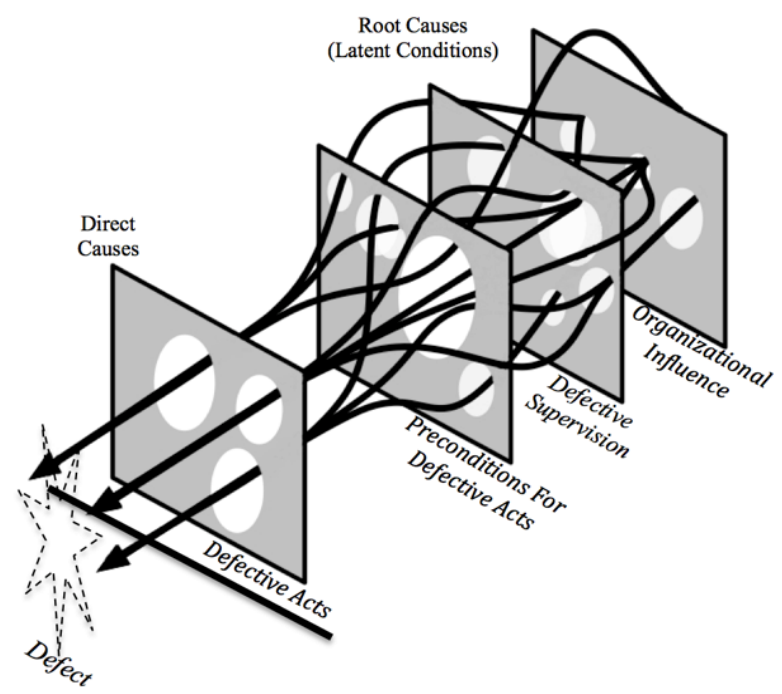

Fig. 1. The Mechanics of Defect Causes (The Swiss Cheese Model).

\section{Methodology AND Data Collection}

In order to identify the latent conditions, this paper involves a combination of a deductive and an inductive approach.

\section{A. Deductive Approach}

The deductive aspect involved the identification of the root causes, attributed to the latent conditions through extensive literature review. The identified latent conditions are classified into each layer in the Swill Cheese model as shown in Table I to Table III.

\section{1) Organizational influences}

Organizational influences (shown in Table I) are decisions made by upper level management that can directly affect practices of the supervisors. These decisions work their way down the Swiss Cheese Model causing the defective act. 
TABLE I: ORGANIZATIONAL INFLUENCES

\begin{tabular}{|c|c|}
\hline Label & Latent Condition \\
\hline L1 & Insufficient liquidity or start-up budget \\
\hline L2 & Organizational Culture \\
\hline L3 & Instable positions of personnel \\
\hline L4 & Allocating unfit or incapable supervisors/engineers for duty \\
\hline L5 & Letting involved in projects that are beyond the organizations \\
\hline L6 & Workplace quality system \\
\hline L7 & Financial constraints upon operational expenses \\
\hline L8 & Time pressure \& constraints \\
\hline L9 & Lack of support from the main office to the site \\
\hline L10 & Lack of motivation/commitment to work \\
\hline L11 & \\
\hline L12 & \\
\hline
\end{tabular}

\section{2) Defective supervision}

At this level the supervisors can influence the conditions of the worker. Inadequate supervision (shown in Table II) in return feeds into the precondition layer.

TABLE II: DEFECTIVE SUPERVISION

\begin{tabular}{|c|c|}
\hline Label & Latent Condition \\
\hline L13 & Change orders* \\
\hline L14 & Failure to correct a known problem \\
\hline L15 & Inadequate supervision \\
\hline L16 & Supervisor/s not adhering to rules or procedures \\
\hline L17 & Poor document control \\
\hline 'L18 & Lack of clear schedule float \\
\hline L19 & Contractor misinterpreted designers' instructions \\
\hline L20 & Designer issued misleading drawings/instructions \\
\hline L21 & Misunderstanding clients requirements \\
\hline L22 & Misleading instructions from worker's direct supervisors \\
\hline L23 & Poor coordination between the project team \\
\hline L24 &
\end{tabular}

\section{3) Preconditions for defective acts}

This layer is the most bottom layer of the latent conditions. It includes condition of the worker, environmental factors and personal factors. Preconditions are usually the most immediate cause of the defective act.

TABLE III: PRECONDITIONS FOR DEFECTIVE ACTS

\begin{tabular}{|c|c|}
\hline Label & Latent Condition \\
\hline L25 & Impaired or poorly maintained tools/machinery \\
\hline L26 & Inappropriate materials supply \\
\hline L27 & Technical/Constructability challenges and constraints \\
\hline L28 & Site Mismanagement \\
\hline L29 & Workers' adverse psychological state (Stress) \\
\hline L30 & Workers' insufficient skill or knowledge level \\
\hline
\end{tabular}

The following section identifies how the root causes were identified and attributed to each of the three latent conditions.

\section{B. Inductive Approach}

The inductive aspect involved conducting a questionnaire with 106 industry professionals to confirm and revise the identified root causes. Respondents from the major job positions were composed of $36 \%$ of project managers, $18 \%$ of site engineers, $11 \%$ of structural engineers, $5 \%$ of mechanical engineers, $4 \%$ of geotechnical engineers, $3 \%$ of traffic engineers, $3 \%$ of architects and $20 \%$ of 'others'. It is crucial to note that $50 \%$ of the respondents had a level of experience of $15+$ years.
The questionnaire was divided into two sections. Section one of the questionnaire asked respondent's to identify a defect instance that was most familiar to them and their perceptions about its causes.

Respondents were provided with a list of 30 latent conditions (Table I to Table III) extracted from the literature. The respondents were asked to indicate, using a six-point Likert scale, to what extent did these root causes contribute to the occurrence of the elected defect. The scale ranged from "Not relevant" for non-existing or un-influential latent conditions, to a highest rate of "Extremely Relevant". Respondents were also provided with an opportunity to identify and rate additional latent conditions that they deemed missing from the list. These additional factors were used to confirm the comprehensiveness of the 30 latent conditions. In most cases, the additional factors were redundant with those identified in the list. In few exceptional cases, the additional factors were held till the end of the survey period and were merged with the pre-identified list. The final lists of latent conditions are same to those provided in Table I to Table III.

Section two of the questionnaire was constructed according to the responses of the first part of the questionnaire. The respondent only had to answer one type of question in section two, "Which of the following factors attributed to the root cause?" The latent conditions would include those identified by the respondents in part 1 . The list of "Factors" available as inputs for each latent condition to choose from was all the other latent conditions that were parallel or in an upper level (Swiss Cheese Model) to that particular root cause (Identified in part 1 of the questionnaire). This resulted in identifying all inputs to a particular root cause. It was assumed that all inputs will only be from the parallel or upper levels of the Swiss Cheese Model, not from the lower levels (i.e., no feedback loops). The number of questions in part two of the survey depended on the number of root causes identified/selected in part one. For example, the selection of 20 of the 30 root causes in part 1 produced 20 questions in part 2. Each of these 20 questions would ask what factors contributed to that particular root cause.

Thenceforth, using the data obtained from section one of the questionnaire, a correlation-matrix (with a majority of 5\%, and few $10 \%$, significance levels) was developed to examine the inter-relationships of the identified causes. The data from section two of the survey was used to establish causality using a two-way Matrix.

\section{Results AnAlysis}

The objective of this study is to determine the inter-relationship between root causes. Correlation and causality was used to establish a relationship between root causes of defects. This section depicts what tools were used to represent the data. This section is followed by the discussion of the results

\section{A. Correlation}

In order to investigate the relationship between root causes, Pearson's Correlation analysis was conducted. The following defect root cause pairs were identified to be highly correlated: 
- Insufficient Liquidity or start-up budget (L1) with Impaired or poorly maintained tools/machinery (L25) (r $=0.808)$.

- Insufficient Liquidity or start-up budget (L1) with Getting involved in projects that are beyond the Organization's Scope (L3) $(r=0.803)$.

- Supervisor/s not adhering to rules or procedures (L12) with Low managerial priority for quality (L7) $(r=0.610)$.

This implies that there exists some connectivity between each of the paired root cause. However correlation does not mean that the relationships between these pairs are essentially direct. Rather, it states that when one root cause was observed to be high so was the other. In order to solidify the relationship between root causes, their inter-causalities were further analyzed

\section{B. Causality}

Inter-causality analysis showed the existence of five strong inputs and seven strong outputs. The strong inputs are described as root causes that are highly influenced. The strong outputs are described as root causes that are highly influential. Highly influential root causes affect all other root causes. While highly influenced root causes are those that are affected.

Root Causes that are Highly Influential include:

- Poor coordination between the project team (L24)

- Designer issued misleading drawings/instructions (L21)

- Time pressure (L10)

- Financial constraints upon operational expenses (L9)

- Organizational culture (L2)

Root Causes that are Highly Influenced include:

- Workers' adverse psychological state (Stress) (L29)

- Impaired or poorly maintained tools/machinery (L25)

- Lack of clear schedule float (L19)

- Lack of support from the main office to the site (L11)

- Change orders (L13)

- Getting involved in projects that are beyond the organisation's capacity (L6)

- Unstable positions of personnel (L3)

From the list above the Root causes that showed the highest and most consistent level of causality were:

- Time pressure (L10)

- Financial constraints upon operational expenses (L9)

- Organizational culture (L2)

The next section discusses the implication of the three root causes with high causality.

\section{DISCUSSION}

This section elaborates on the three most highly influential root causes (i.e. Time pressure, Financial constraints upon operational expenses and Organizational culture). Furthermore, the other causes that are most affected by these will be discussed to elaborate on the possible system improvements that are obtained on the basis of their removal.

\section{A. Time Pressure}

When a project is accelerated, quality is often sacrificed for the sake of remaining ahead of schedule, and the actual schedule benefits may not be worth the time saved [10]. This is what "time pressure" does. The results from this study showed that the following root causes were highly dependent on Time Pressure.

- Lack of clear schedule float (Causality $=0.769$ )

- Workers' adverse psychological state (Stress) (Causality $=0.667$ )

- Supervisor/s not adhering to rules or procedures (Causality $=0.500)$

- Designer issued misleading drawings/instructions (Causality $=0.484)$

The causality value depicts how strong the above four root causes rely on Time Pressure on scale between 0 and 1 .

Stress is negatively related to productivity and efforts have been made to resolve productivity issues related to stress [11], [12]. Time pressure also leads to distortion of the schedule float, which in turn would create stress amongst the workers. The short time frame available will also lead designers to produce drawings in haste leading to misleading instruction. Supervisors trying to meet goal in regards to time will cut corners and take short cuts outside standard procedure. Stress has a contribution towards each one of the four highly dependent root causes. For these reasons, although accelerating a project can be rewarding, the consequences can also be troublesome [13], [14].

\section{B. Financial Constraints upon Operational Expenses}

The root cause "financial constraint" is also of high causality. Individuals usually repeat practices such as taking short cuts not following due processes. When such practices have satisfactory outcomes, as assumed by the individual, then they are utilised on future projects even if it is unsuitable for that project [15]. A few examples of bad practices are the decision by designers to eschew audits, checks, verifications, and reviews prior to releasing documentation for bidding or construction. Despite the importance of such activities, this practice has become a norm due to the financial and time pressures being imposed upon design firms by their clients [3].

The following root causes were highly dependent on the root cause "financial constraints":

- Impaired or poorly maintained tools/machinery (Causality $=0.611$ )

- Workers' adverse psychological state (Stress) (Causality $=0.501$ )

- Time pressure $($ Causality $=0.500)$

Time pressure and stress have already being discussed in the previous section. The lacks of funds intuitively have a strong causality towards poorly maintained machinery or tools. The combination of the above root causes then inevitably leads to defects.

\section{Organizational Culture}

Organizational culture plays a critical role in motivating innovative behavior, as it can create commitment among members of an organization in terms of believing in innovation as an organizational value and accepting innovation-related norms prevalent within the organization [16].

The results from this study showed that the following root causes were highly dependent on Organizational culture.

- Unstable positions of personnel (Causality =0.800) 
- Lack of support from the main office to the site (Causality $=0.667$ )

- Projects: beyond the organization's capacity (Causality $=0.667$ )

Lack of support is an organisational culture in itself. This occurs when contractors' management at the main office do not give enough support to their site managers [2]. This leaves site managers with little direction and support and will cause the project itself to be sidetracked with defects. This may also lead to tendering of project beyond the organisations capacity. This will leave organisation with large projects for which they will lack the tools and the personnel. In order to handle project with large scopes a complicated network of subcontractors need to be hired. This complex level of subcontracting can bring about stress and lack motivation, and will only add to the possibility of defects occurring. Unstable position of personnel has a high causality from organisational culture. It is also highly influenced by "Lack of support from the main office to the site" (Causality $=0.400$ ) and "financial constraints" (Causality $=0.400$ ). These are all input to the "unstable position of the personnel". All these inputs will undoubtedly cause the employees to be in an unstable state, which in turn weakens the control about quality issues.

\section{CONCLUSION}

The literature on the root causes of defects followed by the analysis of data from the field produce a detailed description of root causes of defects in construction. The data was used to demonstrate the inter-relationship between the root causes. This was done and demonstrated in terms of correlation and causality.

This data can be used by practitioners to focus on the root causes with high causality and simultaneously utilize information on correlation of root causes to make the resolving of defects more effective.

This paper showed that the defects originate from different levels of the organization. Most of the defects are from the organizational level. Time pressure, financial constraints upon operational expenses and organizational culture have been identified as the root causes with the highest causality influence. The three root causes above affected most of the other root causes. Accordingly, such root causes can be resolved by focusing efforts to resolve these three causes. The strong connectivity will result in reduction of all root causes therefore reduction of defects.

The most vital aspect of this research paper is that it establishes the root causes that trigger defects. It presents data on the inter-relationship between the root causes of defects. In conclusion it gives practitioners a basis to consider root causes to be an essential aspect in resolving construction defects and reduce cost overruns, accidents, claims and disputes.

\section{REFERENCES}

[1] D. Watt, Building pathology: principles and practice, UK: Blackwell Science Ed, 1999.

[2] P. E. Josephson and Y. Hammarlund, "The causes and costs of defects in construction-A study of seven building Projects," Autom in Constr., vol. 8, no.6, pp. 681-642, October 1999.
[3] P. E. D. Love, D. J. Edwards, Z. Irani, and D. H. T. Walker, "Project pathogens: The anatomy of omission errors in construction and resource engineering projects," IEEE Transactions on Engineering Management, vol. 56, no. 3, pp. 425-435, 2009.

[4] H. Aljassmi and S. Han, "Classification and occurrence of defective acts in residential construction projects," Journal of Civil Engineering and Management, Taylor \& Francis, 2012 (In Press).

[5] H. Aljassmi and S. Han, "Analysis of causes of construction defects using fault trees and risk importance measures", Journal of Construction Engineering and Management, ASCE, 2012 (In Press).

[6] P. F. Wilson, L. D. Dell, and G. F. Anderson, Root Cause Analysis: A Tool for Total Quality Management, ASQC Quality Press, ASQC, Milwaukee, WI, U.S.A, 1993.

[7] J. R. Dew, "In Search of the Root Cause," Quality Progress, vol. 24, no. 3, pp. 97-107, 1991.

[8] B. Sproull, Process Problem Solving: A Guide for Maintenance and Operations Teams, Productivity Press, Portland, 2001.

[9] J. Reason, Human Error, Cambridge University Press, Cambridge, U.K, 1990.

[10] G. Ballard and G. Howell, "Shielding production: An essential step in production control," Journal of Construction Engineering and Management, ASCE, vol. 124, no. 1, pp. 11-17, 1997.

[11] M. F. Dollard, H. R. Winefield, A. H. Winefield, and J. D. Jonge, "Psychosocial job strain and productivity in human service workers: A test of the demand-control support model," Journal of Occupational \& Organizational Psychology, vol. 73, no. 4, pp. 501-510, 2000.

[12] M. Kompier and C. Cooper, "Preventing stress, improving productivity," Routledge, London, 1999.

[13] H. R. Thomas, "Schedule acceleration, work flow, and labour productivity," J. Constr. Eng. Manage., vol. 126, no. 4, pp. 261-267, 2000.

[14] F. Pena-Mora and M. Park, "Dynamic planning for fast-tracking building construction projects," J. Constr. Eng. Manage., vol. 127, no. 6, pp. 445-456, 2001.

[15] J. S. Busby and E. J. Hughes, "Projects, pathogens and incubation periods," International Journal of Project Management, vol. 22, no. 5, pp.425-434, 2004.

[16] A. Hartmann, "The role of organizational culture in motivating innovative behaviour in construction firms," Construction Innovation: Information, Process, Management, vol. 6, no. 3, pp. 159-172, 2006.

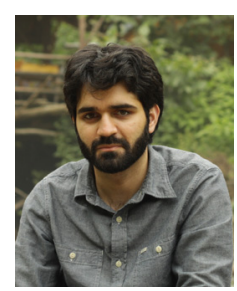

H. Aljassmi received the Bachelors in Civil Engineering degree from the University of Greenwich, U.K. in 2007, and the Masters of Business Administration (MBA) degree from Cardiff University, U.K. in 2009. He is currently pursuing his $\mathrm{PhD}$ in the field of Construction Engineering and Management at the University of New South Wales, Australia. His research interests include defects, errors and rework in construction projects, construction safety, and Building Information Modelling uses and implementation challenges.

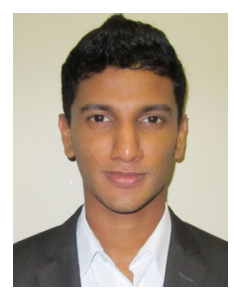

J. Perera received the Bachelors of Engineering from the University of New South Wales, Sydney in 2012 Currently, he is working as an engineer at the civil consultancy firm, Cardno, located at St Leonards, Sydney, Australia. Mr Perera is a member of Engineers Australia. He achieved a class I accolade in 2012 in Engineering at the University of New South Wales. In $2012 \mathrm{Mr}$ Perera was awarded the "Civil Engineering Industrial Training Prize" from the University of New South Wales. He was also involved in the Warrane College, UNSW committee as the President in 2010 and SLSS, UNSW as a ARC delegate in 2009.

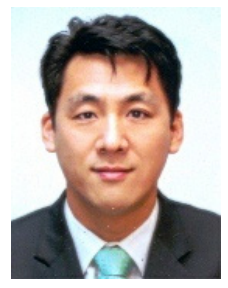

S. Han obtained Ph.D. in the area of Construction Management at the University of Illinios at Urbana-Champaign in 2008. He is currently an Assistant Professor at the University of Seoul. He worked for the School of Civil and Environmental Engineering at the University of New South Wales, Sydney, Australia. His research interests include construction productivity, quality and safety improvement. 